ARTÍCULO DE INVESTIGACIÓN

\title{
Tableros de partículas fabricados con residuos industriales de madera de Pinus patula
}

\section{Particle boards manufactured from industrial residues of Pinus patula wood}

\author{
Luis Zambrano, ${ }^{1}$ Pablo Moreno, ${ }^{2 *}$ Freddy Muñoz, ${ }^{3}$ Jorge Durán, ${ }^{2}$ \\ Darío Garay ${ }^{2}$ y Styles Valero ${ }^{2}$
}

\begin{abstract}
RESUMEN
Este estudio es una propuesta para dar un aprovechamiento y uso a los residuos industriales del aserrío de la madera de Pinus patula para la elaboración de tableros aglomerados de partículas. Por lo tanto, los objetivos fueron manufacturar tableros aglomerados de partículas homogéneos a dos niveles de densidad, determinar sus propiedades físico-mecánicas y evaluar los resultados bajo las normas alemana y venezolana. La absorción de agua promedio a 2 y 24 horas de los tableros a ambas densidades cumplió con lo estipulado en las normas. La variación del espesor (hinchamiento) promedio por inmersión en agua a 2 y 24 horas para los tableros con densidad de $767 \mathrm{~kg} \mathrm{~m}^{-3}$ cumplió con lo exigido en ambas normas. Para el caso de los tableros con densidad de $672 \mathrm{~kg} \mathrm{~m}^{-3}$ la variación de espesor a 24 horas cumplió con las normas, mientras que la variación del espesor a 2 horas no. Respecto de las propiedades mecánicas, tanto el módulo de ruptura en flexión estática como la tensión perpendicular (adhesión interna) para ambos niveles de densidad superaron el valor mínimo aceptado en las normas; demostrando que los residuos industriales de la madera de $P$. patula presentan buenas características tecnológicas para la fabricación de tableros de partículas de densidad media.
\end{abstract}

\section{PALABRAS CLAVE}

Flexión estática, densidad, propiedades físico-mecánicas, tableros de partículas de densidad media, tensión perpendicular, urea-formaldehído.

\begin{abstract}
This study is a proposal to generate uses of industrial residues from sawmills of Pinus patula wood for the manufacturing of particleboards. The aims are to manufacture homogeneous particleboards with two density levels, determine their physical and mechanical properties and evaluate the results with German and Venezuelan standards. The average water absorption at 2 and 24 hours of both density levels of boards meets the requirements for both standards. The average thickness variation (swelling) by immersion en water at 2 and 24 hours for boards with average density of $767 \mathrm{~kg} \mathrm{~m}^{-3}$ meets the requirements for both standards. For the case of the panels with average density of $672 \mathrm{~kg}$ $\mathrm{m}^{-3}$ thickness variation at 24 hours meets the requirements of both standards, while the swelling at 2
\end{abstract} hotmail.com

2 Universidad de Los Andes, Facultad de Ciencias Forestales y Ambientales. Laboratorio Nacional de Productos Forestales. Mérida-Venezuela. C.e.: jorduran@ula.ve, dargaray@ula.ve,styles@ula.ve

3 Tecnológico de Costa Rica (TEC). Escuela de Ingeniería Forestal. Centro de Investigación en Integración Bosque-Industria (CIIBI). Cartago-Costa Rica. C.e.: fmunoz@tec.ac.cr

Autor para correspondencia: C.e.: pmoreno@ula.ve 
hours does not. Regarding the mechanical properties both the modulus of rupture in static bending and tension perpendicular (internal bond) for both real density levels exceed the minimum accepted by standards, demonstrating that industrial residues from $P$. patula wood exhibit good technological characteristics for medium density particleboards manufacture.

\section{KEY WORDS}

Static bending, density, physical and mechanical properties, medium density particleboard, perpendicular tension, urea-formaldehyde.

\section{INTRODUCCIÓN}

Para un buen desarrollo de la industria forestal y maderera venezolana es imprescindible el aprovechamiento integral de la madera, optimizando el rendimiento de los recursos maderables, es decir, utilizando en los procesos productivos el mayor porcentaje posible de la madera cosechada del bosque. Tradicionalmente, en Venezuela se aprovechan solamente las trozas provenientes del fuste principal hasta una altura comercial aproximada de $15 \mathrm{~m}$, lo cual implica la pérdida de un volumen considerable de madera que incluye trozas de altura superior, copa y ramas. De igual manera, los residuos producidos por el procesamiento primario en las industrias madereras no son aprovechados, generando un volumen considerable de subproductos de material lignocelulósico, que particularmente en Venezuela no han sido aprovechados, debido a la existencia de recursos maderables abundantes y falta de políticas de aprovechamiento integral de los subproductos generados del procesamiento forestal industrial. Sin embargo, estos subproductos pueden ser utilizados en la fabricación de otros productos de ingeniería con múltiples aplicaciones.

Según lo señalan Alma et al., (2004), la población del mundo consume actual- mente más de 3,5 millones de toneladas de madera verde al año. Si la tasa de consumo de fibra de madera y la tasa de crecimiento de la población (de aproximadamente 90 millones de personas por año) se mantienen constantes, la demanda de fibra de madera se incrementará en más de 60 millones de toneladas cada año, lo que podría aumentar la tasa de deforestación (Zheng et al., 2006), propiciando el agotamiento de algunas especies forestales de bosque natural. Aprovechar de manera óptima e integral el recurso forestal, los residuos industriales, las especies de rápido crecimiento y las fibras no leñosas, como los residuos agrícolas (bagazo y paja de trigo), es una buena opción para mitigar y disminuir presiones políticas y sociales, sobre la explotación de maderas procedentes de bosque natural (Latibari y Roohnia, 2010).

Un producto de ingeniería que se adapta a los requerimientos anteriormente mencionados son los tableros de partículas, pues el suministro de materia prima para su elaboración potencia el uso de subproductos originados por las operaciones forestales (podas y raleos) hasta los del proceso primario y secundario de la madera (despuntes, descuadres, costaneras, corteza, entre otros). De esta manera se generan nuevas alternativas para promover el desarrollo de la industria maderera venezolana con nuevos productos de ingeniería con diferentes aplicaciones.

Existen diversos antecedentes bibliográficos referidos al uso exitoso de residuos provenientes de la industria forestal y maderera en la elaboración de tableros aglomerados de partículas, como ejemplo están las investigaciones realizadas por (Ginzel y Peraza, 1966; Moslemi, 1974; Peredo y Deppe, 1984; Suchsland y Xu, 1989; Poblete y Sánchez, 1991; Peredo y Torres, 1991; Van Niekerk y Pizzi, 1994; Blanchet et al., 2000; Moreno et al., 2005; Pedieu et al., 2008; Latibari y Roohnia, 
2010; Bazyar et al., 2011). Por otro lado, Garay et al. (2009), estiman que las propiedades de un tablero están determinadas por los siguientes factores: tipo, tamaño, distribución y orientación de las partículas, así como también el tipo y la calidad del proceso, contenido de humedad durante el prensado y tratamiento post-manufactura del tablero.

El presente estudio es una propuesta sobre el aprovechamiento y uso de los residuos industriales del aserrío de la madera de $P$. patula para la elaboración de tableros aglomerados de partículas.

\section{OBJETIVOS}

Los objetivos fueron: a) manufacturar tableros aglomerados de partículas homogéneos a dos niveles de densidad, b) determinar sus propiedades físico-mecánicas y c) evaluar los resultados de acuerdo con lo establecido en la norma alemana DIN (1981) y venezolana COVENIN (1991).

\section{MATERIALES Y MÉTODOS}

Como materia prima se utilizaron costaneras derivadas del proceso de aserrío de árboles de $P$. patula de 25 años de edad, procedentes del bosque La CarboneraSan Eusebio, Mérida-Venezuela. La elaboración de los tableros, la preparación de probetas y la ejecución de los ensayos, se realizaron en el Laboratorio Nacional de Productos Forestales: Sección tableros aglomerados de la Facultad de Ciencias Forestales y Ambientales de la Universidad de Los Andes, Venezuela.

\section{Determinación de la densidad y contenido de humedad de la materia prima}

De los segmentos de costaneras seleccionadas al azar, se prepararon 30 probetas con dimensiones de $30 \mathrm{~mm} \times 30 \mathrm{~mm} \times 100$ $\mathrm{mm}$ para la determinación de la densidad en la condición verde, seca al aire y seca al horno, según la norma DIN (1976). El cálculo del contenido de humedad se realizó con la siguiente ecuación:

$$
\% C H=\left(\frac{P_{i}-P_{o}}{P_{o}}\right) \cdot 100
$$

donde:

$\% \mathrm{CH}=$ contenido de humedad.

$\mathrm{p}_{\mathrm{i}}=$ peso inicial de la muestra $(\mathrm{g})$.

$p_{o}=$ peso de la muestra secada al horno (g).

\section{Viruteado de los residuos de $P$. patula y secado de partículas}

Las costaneras fueron procesadas en una viruteadora de cuchillos para obtener partículas con una dimensión promedio de 20 $\mathrm{mm}$ de longitud, ancho variable y espesor de $0,25 \mathrm{~mm}$. Desde la viruteadora las partículas fueron transferidas por un sistema neumático de ciclones a la secadora de suspensión Keller, donde fueron secadas a un contenido de humedad final de $4 \%$.

\section{Coeficiente de esbeltez}

De manera aleatoria se tomaron $30 \mathrm{~g}$ de las partículas secas para determinar su geometría y tamaño, éstas se clasificaron de manera visual en tres categorías de tamaño: grandes, medianas y pequeñas. Cada categoría fue pesada y se tomaron 30 muestras de cada una. Con un vernier digital de $0,02 \mathrm{~mm}(0,0001$ pulgadas $)$ de resolución, se midió largo, ancho y espesor de las partículas y se determinó su coeficiente de esbeltez $(E)$, con la ecuación (2). La proporción de participación de cada categoría de tamaño de partícula para conformar el tablero, se determinó mediante su peso. 


$$
E=\left(\frac{L}{e}\right)
$$

donde:

$E=$ coeficiente de esbeltez.

$L=$ longitud de las partículas $(\mathrm{mm})$.

$e=$ espesor de las partículas $(\mathrm{mm})$.

\section{Componentes para la fabricación de los tableros}

Se utilizaron los siguientes insumos: partículas de $P$. patula a $4 \%$ de contenido de humedad, adhesivo urea formaldehído (UF) a $60 \%$ de sólidos con viscosidad = $250 \mathrm{cP}, \mathrm{pH}=8,5$ y como catalizador una solución de sulfato de amonio al 30\% (p/p) de concentración, la cantidad de catalizador corresponde al $8 \%(\mathrm{p} / \mathrm{p})$ de la cantidad de adhesivo aplicada a cada tablero. Se fabricaron cinco tableros homogéneos para cada nivel de densidad.

\section{Encolado de las partículas}

El encolado de las partículas secas a $4 \%$ se realizó por el método de aspersión a $0,203 \mathrm{MPa}$ (2 atm) por un tiempo de 8 minutos en una encoladora Drais usando una carga de adhesivo UF catalizado de $12 \%$ respecto al peso seco de las partículas.

\section{Formación del colchón, proceso de pre-prensado y prensado del tablero de partículas}

Luego de encoladas las partículas fueron distribuidas, manualmente, sobre una lámina de aluminio previamente parafinada y humectada con agua. Para retener las partículas en todos los costados del colchón se utilizó un marco o formaleta de madera. Una vez conformado el colchón de partículas se aplicó una carga de aproximadamente $785 \mathrm{~N}(80 \mathrm{~kg})$ a temperatura ambiente con la finalidad de compactar y disminuir el espesor, evitando la deformación del tablero en el proceso de prensado. Finalmente, para obtener los dos niveles de densidad en los tableros de partículas se aplicaron los respectivos ciclos de prensado (Fig. 1) en una prensa Becker van Huller bajo las siguientes condiciones operacionales: presión específica sobre el tablero: 3,05 MPa $\left(31,14 \mathrm{~kg} \mathrm{~cm}^{-2}\right)$, presión manométrica de la prensa simple: $14,71 \mathrm{MPa}$ (150 $\mathrm{kg} \mathrm{cm}^{-2}$ ), temperatura de los platos de la prensa: $180^{\circ} \mathrm{C}$ a $200^{\circ} \mathrm{C}$, tiempo de prensado total: 9 min para densidad teórica $650 \mathrm{~kg} \mathrm{~m}^{-3}$ y 18 min para densidad teórica $750 \mathrm{~kg} \mathrm{~m}^{-3}$.

\section{Acondicionamiento y dimensionado de los tableros}

Los tableros fabricados fueron almacenados en un cuarto de acondicionamiento a $20{ }^{\circ} \mathrm{C} \pm 1{ }^{\circ} \mathrm{C}$ y una humedad relativa de $65 \% \pm 2 \%$. Los tableros permanecieron bajo esas condiciones hasta que alcanzaron un contenido de humedad de equilibrio de $12 \%$ aproximadamente. Luego los tableros fueron dimensionados en una sierra circular con escuadría de $500 \mathrm{~mm}$ x $500 \mathrm{~mm}$, conservando el espesor de prensado de $19 \mathrm{~mm}$.

\section{Ensayos físicos y mecánicos de los tableros}

A partir de los tableros dimensionados se obtuvieron 10 probetas para cada uno de los ensayos, según las normas DIN (1965 b, c, dye). Los ensayos físicos fueron: contenido de humedad, densidad del tablero, absorción por inmersión en agua y variación de espesor por inmersión en agua a 2 y 24 horas. Los ensayos mecánicos fueron: resistencia a flexión estática (módulo de ruptura, MOR) y tensión per- 


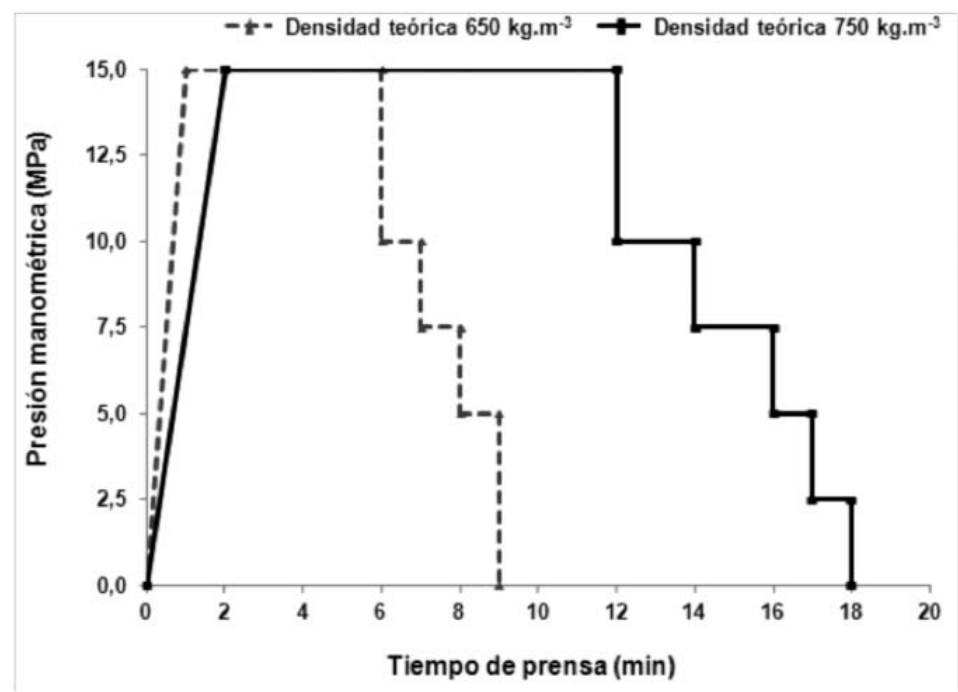

Figura 1. Diagrama del ciclo de prensado aplicado para densificar los tableros homogéneos de partículas de Pinus patula a las densidades teóricas propuestas.

pendicular a la superficie del tablero (adhesión interna) usando una máquina universal Instron y una velocidad de ensayo de $5 \mathrm{~mm} / \mathrm{min}$ para ambos ensayos. La evaluación de las propiedades físico-mecánicas de los tableros se realizó según lo estipulado por la norma venezolana COVENIN (1991) y alemana DIN (1981), utilizadas para tableros de partículas de madera de uso general con prensado plano.

\section{Diseño experimental}

Se utilizó un diseño con un sólo factor (densidad) con dos niveles categóricos y seis variables respuesta (absorción de agua a 2 h y 24 h, variación de espesor o hinchamiento a 2 h y 24 h, módulo de ruptura en flexión estática y tensión paralela a la superficie del tablero).

El análisis de varianza (ANDEVA) se realizó con un nivel de confianza $\alpha=0,05$ y con el software estadístico Design Expert $^{\circledR} \mathrm{V}$ 8.0.

\section{RESULTADOS}

\section{Análisis de varianza (ANDEVA) de un solo factor}

EI ANDEVA (Tabla 1) indica que el factor densidad del tablero es significativo para todas las variables respuesta. La probabilidad normal de residuales de los datos obtenidos en todas las variables respuesta cumple con el supuesto de normalidad, no se observaron fluctuaciones significativas de las muestras ensayadas ni datos atípicos. La proporción de variabilidad de cada parámetro respuesta es explicada por el modelo estadístico mediante el valor de $R^{2}$. Por otra parte, el valor $R^{2}$ ajustado indica que el número de factores presentes en el modelo es adecuado, por lo tanto, ambos confirman la validez del ANDEVA.

En la figura 2 se modela el efecto del factor densidad sobre las propiedades físico-mecánicas determinadas para los tableros aglomerados de $P$. patula. 
Tabla 1. Valor-p para las variables respuesta.

\begin{tabular}{ccccccc}
\hline & \multicolumn{5}{c}{ Valor- $p^{*}(\alpha=0,05)$} \\
\cline { 2 - 6 } Factor & ABS 2h & ABS 24h & VE 2h & VE 24h & MOR & TPST \\
\hline \multirow{2}{*}{ Modelo } & $<0,0001$ & $<0,0001$ & 0,0002 & 0,0022 & $<0,0001$ & 0,0002 \\
Densidad & $<0,0001$ & $<0,0001$ & 0,0002 & 0,0022 & $<0,0001$ & 0,0002 \\
R2 & 0,9964 & 0,9881 & 0,8371 & 0,7103 & 0,9768 & 0,8347 \\
R2 ajustado & 0,9959 & 0,9867 & 0,8167 & 0,6741 & 0,9739 & 0,8140 \\
\hline
\end{tabular}

FUENTE: software estadístico Design Expert ${ }^{\circledR} \vee 8.0$

$\mathrm{ABS}=$ absorción de agua a 2 y 24 h; VE= variación de espesor a 2 y 24 h; MOR= módulo de ruptura en flexión estática; TPST = tensión perpendicular a la superficie del tablero

* Valor- $p<0,05$ indica que los términos del modelo son significativos.

* Valor- $p>0,05$ indica que los términos del modelo no son significativos.

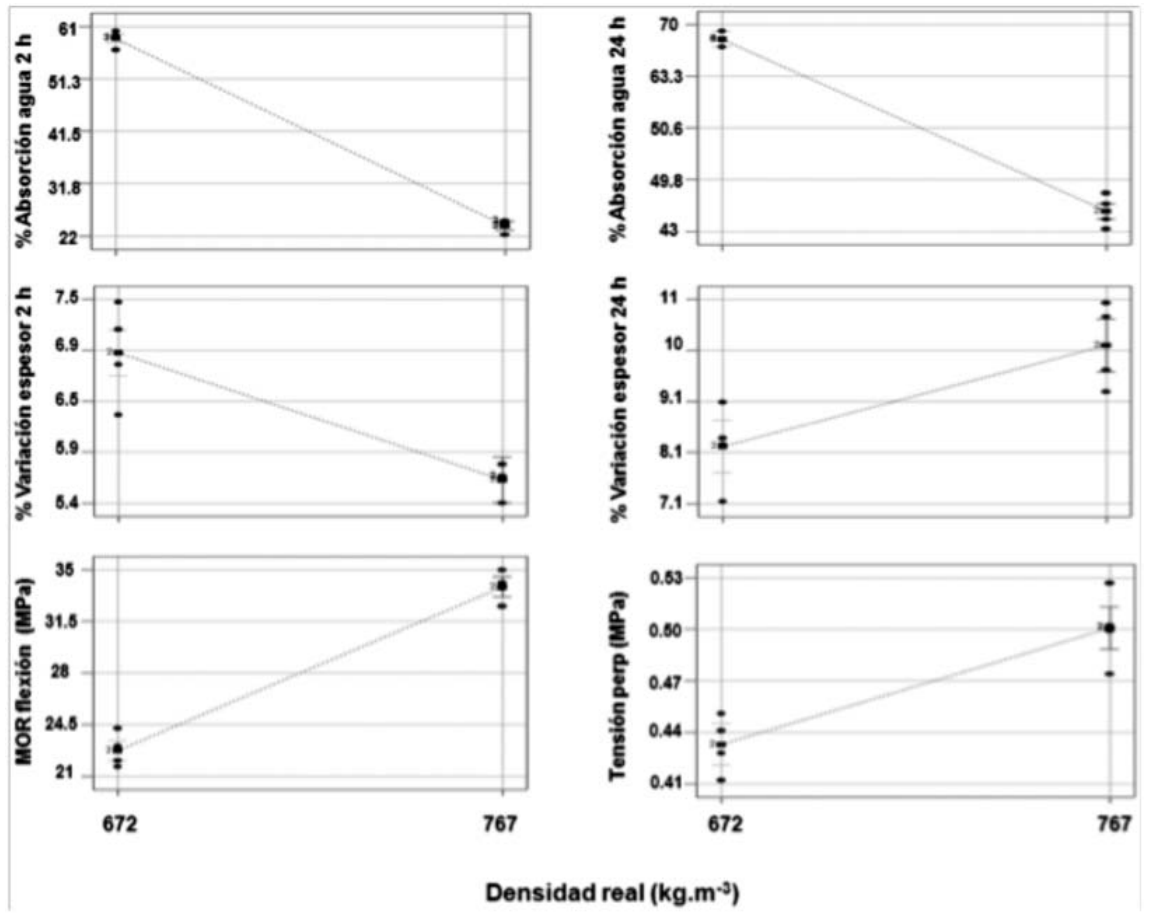

Figura 2. Modelamiento con software Design Expert ${ }^{\circledR}$ del efecto del factor densidad sobre las propiedades físico-mecánicas de los tableros homogéneos de Pinus patula. 


\section{Densidad de la madera de $P$. patula y tableros de partículas}

Los valores de la densidad promedio obtenidos para madera sólida utilizada como materia prima para la fabricación de tableros aglomerados de partículas, se presentan en la tabla 2. Los intervalos de densidad y coeficientes de variación para los tableros se muestran en la tabla 3 .

Una comparación entre la densidad teórica y la real de los tableros de partículas y madera sólida de $P$. patula se presenta en la figura 3.

\section{Coeficiente de esbeltez}

Los valores promedio de participación de cada intervalo de partículas de $P$. patula seleccionado y su respectivo coeficiente de esbeltez se muestran en la tabla 4.

El valor promedio del coeficiente de esbeltez para las partículas de $P$. patula $(148,92)$ se encuentra dentro del intervalo de esbeltez comprendido entre 120 y 200, intervalo que es considerado por Moslemi (1974), Medina (1975), Peredo (1988), Garay (1997) y Moreno et al. (2002), como óptimo para la elaboración de tableros de partículas con propiedades físicas y mecánicas aceptables.

Tabla 2. Densidad promedio para la madera sólida de Pinus patula utilizada para la fabricación de tableros aglomerados de partículas.

\begin{tabular}{cccc}
\hline Condición & Densidad promedio $\left(\mathrm{kg} \mathrm{m}^{-3}\right)$ & $\begin{array}{c}\text { Coeficiente de } \\
\text { variación (\%) }\end{array}$ & $\begin{array}{c}\text { Contenido de } \\
\text { humedad (\%) }\end{array}$ \\
\hline Verde & 684 & 4,4 & 30,50 \\
Seca al aire & 668 & 6,2 & 13,31 \\
Seca al horno & 618 & 5,3 & ------ \\
\hline
\end{tabular}

Tabla 3. Densidad promedio y coeficiente de variación de los tableros aglomerados de partículas de Pinus patula.

\begin{tabular}{cc}
\hline $\begin{array}{c}\text { Densidad real promedio } \\
(\mathrm{kg} \mathrm{m}-3)\end{array}$ & $\begin{array}{c}\text { Coeficiente de variación } \\
(\%)\end{array}$ \\
\hline 672 & 3,25 \\
767 & 2,88 \\
\hline
\end{tabular}




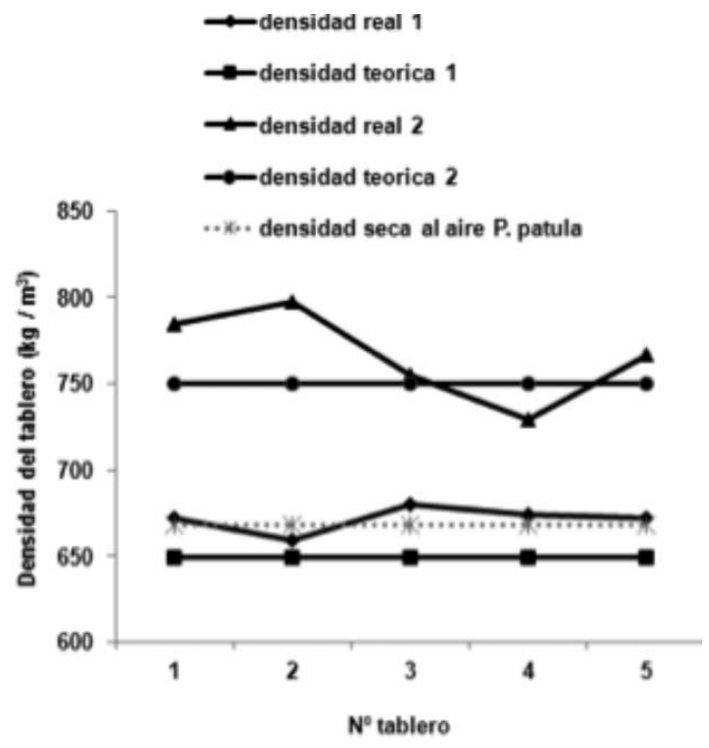

Figura 3. Comparación entre densidad teórica y real obtenida para los tableros homogéneos de partículas y la densidad de la madera sólida seca al aire de Pinus patula.

Tabla 4. Coeficiente de esbeltez $(E)$ de las partículas de Pinus patula.

\begin{tabular}{ccccc}
\hline $\begin{array}{c}\text { Rango de tamaño en ancho } \\
(\mathrm{mm})\end{array}$ & $\begin{array}{c}\text { Proporción en } \\
\text { peso }(\%)\end{array}$ & $\begin{array}{c}\text { Largo } \\
(\mathrm{mm})\end{array}$ & $\begin{array}{c}\text { Espesor } \\
(\mathrm{mm})\end{array}$ & $E$ \\
\hline 18 & 60,00 & 41,07 & 0,23 & 178,56 \\
$9-18$ & 27,50 & 29,62 & 0,22 & 134,63 \\
$<9$ & 12,50 & 29,39 & 0,22 & 133,59 \\
\hline Promedio & 100 & 26,69 & 0,22 & 148,92 \\
\hline
\end{tabular}

\section{Absorción de agua por inmersión}

En las figuras $4 a$ y $4 b$, se presenta el comportamiento de la absorción de agua, por inmersión a 2 y 24 horas, de los tableros a ambas densidades y de acuerdo con la norma COVENIN (1991) que solicita $25 \%$ y $60 \%$ de absorción para 2 horas y 24 horas, respectivamente. Los tableros con densidad de $672 \mathrm{~kg}^{-3} \mathrm{y}$ absorción de agua a 2 y 24 horas no cumplen con lo solicitado por la norma. Mientras que, los tableros con densidad de $767 \mathrm{~kg} \cdot \mathrm{m}^{-3}$ sí lo cumplen. 


\section{Variación de espesor por inmersión en agua}

En las figuras $4 c$ y $4 d$, se presenta el comportamiento de la variación de espesor por inmersión en agua a 2 y 24 horas de los tableros con ambas densidades y de acuerdo con las normas COVENIN (1991) y DIN (1981) que solicitan una variación no mayor a $6 \%$ y $15 \%$ a 2 horas y 24 horas, respectivamente. Los tableros con densidad de $672 \mathrm{~kg} \cdot \mathrm{m}^{-3}$ no cumplen con la exigencia de la norma COVENIN (1991) para la variación de espesor por inmersión en agua a 2 horas, pero sí cumplen los requerimientos para la variación de espesor a 24 horas.

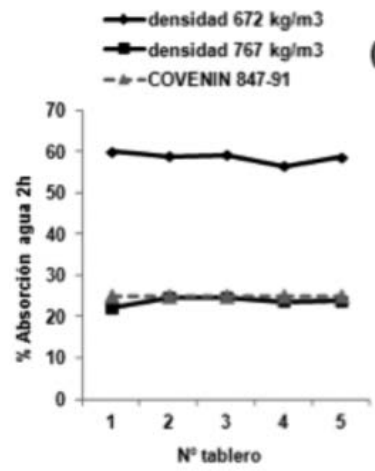

Los tableros con densidad de 767 $\mathrm{kg} \cdot \mathrm{m}^{-3}$ sí cumplen con lo solicitado por las normas para la variación de espesor por inmersión en agua a 2 y 24 horas.

\section{Flexión estática (MOR)}

En la figura $5 a$, se presentan los valores del MOR en flexión estática de los tableros de $P$. patula para ambas densidades. Los tableros con densidad de $672 \mathrm{~kg} \cdot \mathrm{m}^{-3}$ y 767 $\mathrm{kg} \cdot \mathrm{m}^{-3}$ superan en $23 \%$ y $52 \%$, respectivamente, al valor mínimo de 17,16 MPa (175 $\mathrm{kg} \mathrm{cm}^{-2}$ ) establecido por las normas COVENIN (1991) y DIN (1981).
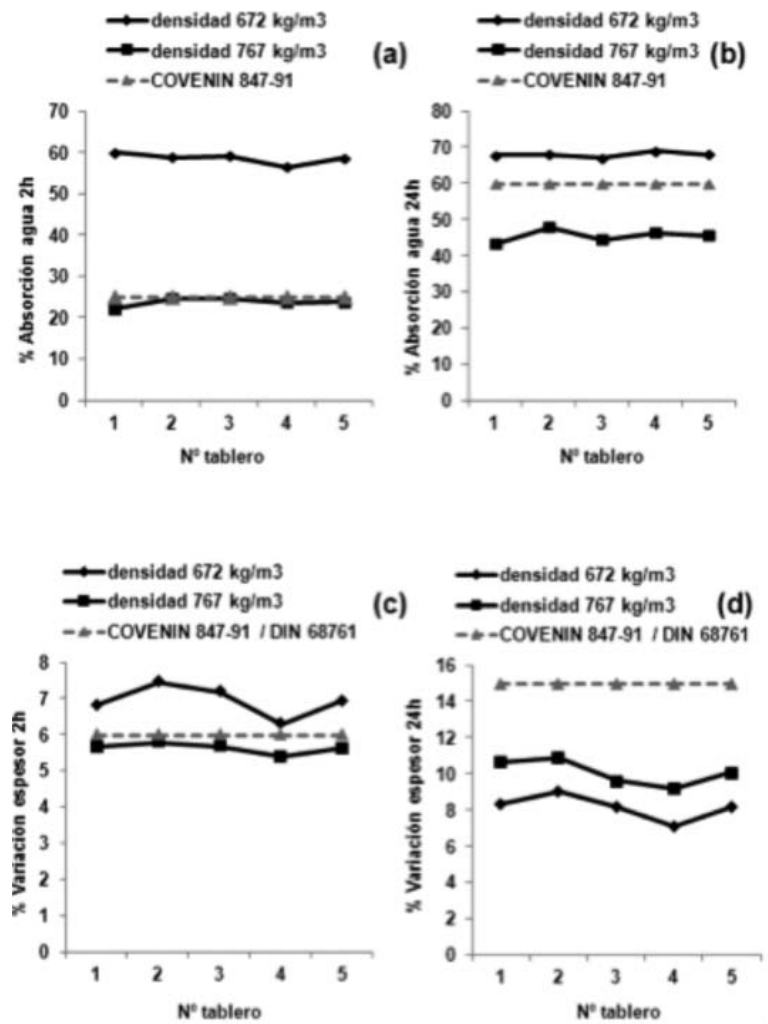

Figura 4. Absorción de agua por inmersión (a) 2 h (b) 24 h y variación de espesor o hinchamiento por inmersión en agua (c) 2 h (d) 24 h. Para tableros homogéneos de partículas de Pinus patula. De acuerdo a las normas COVENIN (1991) y DIN (1981). 
Tensión perpendicular a la superficie del tablero

En la figura $5 b$ se presentan los valores obtenidos para la tensión perpendicular a la superficie de los tableros de P. patula para ambas densidades. Los tableros con densidad de $672 \mathrm{~kg} \cdot \mathrm{m}^{-3}$ y $767 \mathrm{~kg} \cdot \mathrm{m}^{-3}$ superan en $19 \%$ y $30 \%$, respectivamente, al valor mínimo de 0,34 MPa $\left(3,5 \mathrm{~kg} \mathrm{~cm}^{-2}\right)$ solicitado por las normas COVENIN (1991) y DIN (1981).

\section{DISCUSIÓN}

El ciclo de prensado permitió obtener tableros con valores similares a la densidad de la madera seca al aire de P. patula. Sin embargo, aun cuando en la conformación del producto participe una sola especie, la variabilidad de la densidad dentro y entre tableros es posible, situación que menciona en su investigación Moreno et al. (2005) con tableros de partículas de ramas de Fraxinus americana. No

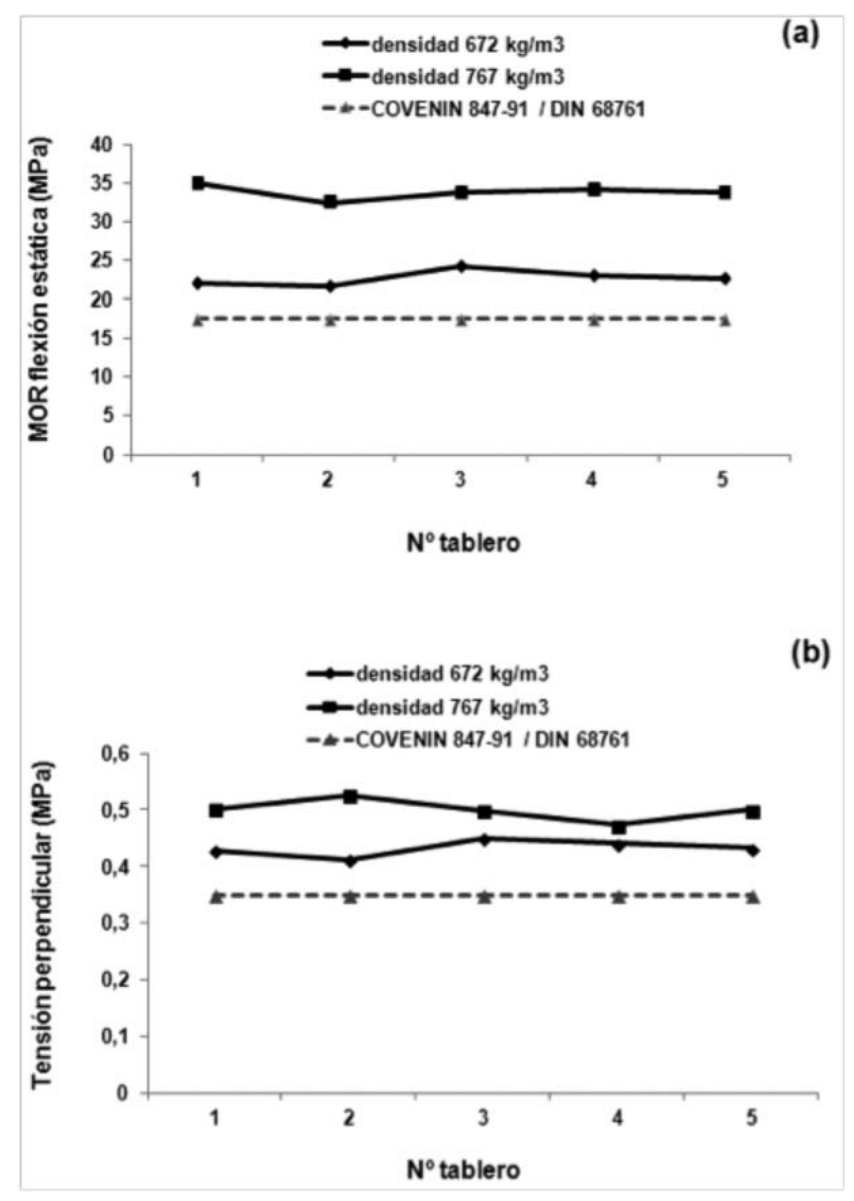

Figura 5. (a) Módulo de ruptura (MOR) promedio en flexión estática y (b) adhesión interna promedio para tableros homogéneos de partículas de Pinus patula. De acuerdo a normas COVENIN (1991) y DIN (1981). 
obstante, los coeficientes de variación determinados para la densidad de los tableros de $P$. patula indican una buena distribución de las partículas en el producto final, de acuerdo con lo propuesto por Durán (1981).

La densidad del tablero es una medida de la compactación individual de una partícula dentro del tablero y es dependiente, principalmente, de la densidad de la madera y la presión aplicada durante el prensado (Rocha et al., 1974). Un incremento en la densidad del tablero resulta en una mejor eficiencia de la resina, debido a la mejora de uniones adhesivas entre partícula y adhesivo (Gatchell et al., 1966). Por ejemplo, Poblete y Peredo (1990) lograron establecer, para tableros de partículas con especies nativas chilenas, ecuaciones de regresión lineal entre la propiedad de flexión y densidad, logrando producir tableros de partículas con densidad de $650 \mathrm{~kg} \cdot \mathrm{m}^{-3}$ que cumplieron con el valor mínimo de 15,99 MPa (163 kg cm${ }^{-2}$ ) solicitado por la norma DIN (1965).

De acuerdo con Rocha et al. (1974), la densidad de la madera de la especie influye en el consumo de adhesivo y la rugosidad superficial del tablero. El usar especies que tengan una alta densidad provoca una disminución de la resistencia mecánica del tablero ya que habría poca tasa de compresibilidad de las partículas, provocando el aumento de la expansión lineal e hinchamiento del espesor en el tablero.

Independiente del tiempo de inmersión, el incremento de la densidad del tablero produce una disminución en la absorción de agua, comportamiento que señalan (Rocha et al., 1974; Durán, 1982; Poblete y Peredo, 1990; Moreno et al., 2002) para tableros de partículas. Esta tendencia se debe a que una mayor densificación produce una disminución en la porosidad del tablero, menor cantidad de espacio vacío entre partículas y adhesivo, lo que impide la penetración del agua. Así lo demuestran las investigaciones de Durán (1981 y 1982); Poblete y Peredo (1990); Maloney (1993) y Moreno et al. (2005), quienes indican que la facilidad de absorción de agua está en función de la relación de compresibilidad del tablero, eficiencia del adhesivo y la facilidad de penetración del fluido (permeabilidad del tablero) por los espacios existentes entre partículas. Relaciones de compresibilidad bajas permiten al tablero absorber más humedad en comparación con tableros con alta tasa de compresibilidad.

Los tableros encolados con adhesivo UF no son aptos para uso a la intemperie (Peredo y Torres, 1991) una exposición prolongada a la humedad lleva a la ruptura de los enlaces que forman la adhesión entre los grupos activos de la madera (partículas) y el adhesivo (Stark et al., 2010) afectando sus propiedades físicomecánicas. Un calor excesivo también produce una degradación química del fraguado de la resina UF, por lo que, típicamente los tableros son enfriados rápidamente luego de finalizado el ciclo de prensado (Stark et al., 2010).

La variación de espesor para los tableros de baja densidad se debe a la facilidad de penetración del agua en los capilares y espacios entre partículas, originando un mayor hinchamiento de la masa de partículas a este nivel de densidad. En los tableros de mayor densidad existe un mayor grado de compresibilidad, contribuyendo a disminuir el grado de higroscopicidad de las partículas de madera.

Los porcentajes de variación en espesor después de 24 horas de inmersión obtenidos para los tableros de $P$. patula son similares a los encontrados por Moreno et al. (2005) en tableros con partí- 
culas de F. americana a dos niveles de densidad $\left(600 \mathrm{~kg} \cdot \mathrm{m}^{-3}-800 \mathrm{~kg} \cdot \mathrm{m}^{-3}\right)$ y por Guler et al. (2008) en tableros de partículas de Pinus nigra con una densidad de $700 \mathrm{~kg} \cdot \mathrm{m}^{-3}$.

Por otra parte, los resultados aquí obtenidos para la variación de espesor a 24 horas de inmersión están en concordancia con lo que exponen Durán (1981) y Moreno et al. (2005 y 2010) que, a mayor densidad del tablero, mayor será el hinchamiento; pero es contario a lo mencionado por Poblete y Peredo (1990) quienes no encontraron una correlación adecuada entre hinchamiento y densidad del tablero.

Aumentar el tiempo de inmersión a 24 horas permite saturar paredes celulares que por su bajo contenido de humedad tienen capacidad de absorción produciendo un hinchamiento en las partículas, manifestándose finalmente en la variación del espesor del tablero. Como se ha mencionado anteriormente existe variación de la densidad dentro del tablero, por lo que el hinchamiento del tablero tampoco será uniforme, es decir, el tablero se deforma y pierde estabilidad dimensional.

El hinchamiento del espesor está directamente afectado por muchas variables de proceso, pero las interacciones entre las variables pueden cambiar este comportamiento, por ejemplo, la durabilidad del tablero depende de hinchamientos bajos siendo una práctica aplicar un alto contenido de resina adhesiva para controlar el hinchamiento del tablero. Sin embargo, Halligan (1970), menciona que varias investigaciones sobre la eficiencia de los tratamientos para el control del hinchamiento han dado resultados contradictorios y propone que un vaporizado post-tratamiento parece ser una opción prometedora. Al respecto, Peredo y Torres (1991), indican en su investigación que la aplicación de agentes hidrófobos reduce el efecto negativo de la humedad sobre la resina UF, disminuyendo significativamente la absorción de humedad e hinchamiento del tablero de partículas y en consecuencia mejorando las propiedades mecánicas del mismo.

Gatchell et al. (1966), así como Roffael y Rauch (1972), exponen que incrementando la densidad del tablero podrían esperarse mayores hinchamientos; sin embargo, de acuerdo con Lehmann (1960), el beneficio de obtener aumento de la resistencia mecánica es razón suficiente para compensar la tendencia de hinchamiento.

El módulo de ruptura es aceptado como una medida de esfuerzo y representa la carga máxima de un miembro que sometido a flexión llega a su fractura (Cai y Ross, 2010). Sin embargo, la resistencia al esfuerzo en flexión debe ser considera hasta el límite elástico, para efectos de seguridad (McNatt, 1973). Mientras que, la adhesión interna o tensión perpendicular determina la resistencia del tablero a la delaminación o separación de sus capas (Cai y Ross, 2010).

El MOR en flexión estática se incrementa a mayor densidad del tablero, esto se debe entre otros factores a una mayor eficiencia en la distribución de la resina y a un mejor contacto entre partículas-adhesivo, así como también a una mayor densificación de las partículas (mayor razón de compresibilidad), disminuyendo el volumen de espacios vacíos. Esto conlleva a que el tablero de mayor densidad trasmita los esfuerzos de partícula a partícula de manera más uniforme traduciéndolos en un aumento de la resistencia mecánica (Durán, 1981; Maloney, 1993 y Bazyar et al., 2011).

En general, el MOR en flexión obtenido en este estudio con los tableros de 
ambas densidades (Fig. 5a) supera al intervalo de MOR de 15,20 MPa - 21,97 MPa (155 kg cm-2 - $224 \mathrm{~kg} \mathrm{~cm}^{-2}$ ) encontrado por Cai y Ross (2010) para tableros de partículas tipo industrial con densidad de $690 \mathrm{~kg} \mathrm{~m}^{-3}$.

En cuanto a la tensión perpendicular a la superficie del tablero (TPST), aumenta a medida que se incrementa la densidad del tablero y la relación de compresibilidad. Esto se puede atribuir al mayor contacto relativo entre las partículas y la mayor eficiencia de la resina, así como también a que se logra una densificación mayor en el centro del tablero debido al mayor volumen de partículas a ser prensadas. Por lo tanto, el gradiente de densidad en el espesor del tablero es menos variable que en el caso de los tableros de menor densidad (Durán, 1982; Maloney, 1993 y Moreno et al., 2002).

En general, la adhesión interna obtenida en este estudio con los tableros de ambas densidades está dentro de los intervalos mencionados por Cai y Ross (2010), de 0,314 MPa - 1,177 MPa (3,2 kg $\mathrm{cm}^{-2}-12,0 \mathrm{~kg} \mathrm{~cm}^{-2}$ ) para tableros de partículas de tipo industrial con densidad de $690 \mathrm{~kg} \cdot \mathrm{m}^{-3}$, y el intervalo de 0,314 $\mathrm{MPa}-$ $0,490 \mathrm{MPa}\left(3,2 \mathrm{~kg} \mathrm{~cm}^{-2}-5,0 \mathrm{~kg} \mathrm{~cm}^{-2}\right)$ encontrado por Guler et al. (2008) para tableros de partículas con una densidad de $700 \mathrm{~kg} \mathrm{~m}^{-3}$ fabricados con residuos de Pinus nigra.

Finalmente, el modelamiento con el software estadístico Design Expert con una confiabilidad de $95 \%$ indica que, a mayor densidad, disminuye la absorción de humedad a ambos tiempos de inmersión, aumenta el hinchamiento o variación del espesor a 24 horas de inmersión. Los esfuerzos en flexión estática y tensión perpendicular (adhesión interna) también aumentan. Estos resultados son coincidentes con lo presentado en las figuras 4 y 5 .

\section{CONCLUSIONES}

Como se demostró en el análisis de varianza y en los resultados obtenidos, la densidad del tablero es un factor que influye de manera directa sobre las propiedades físico-mecánicas de un tablero aglomerado de partículas.

El aprovechamiento de los residuos industriales de la especie Pinus patula, mostró buenos resultados y propiedades tecnológicas adecuadas para su trasformación en un producto de ingeniería como el tablero homogéneo de una capa de partículas con densidad media. Los tableros de este estudio cumplieron con los valores mínimos solicitados por las normas, en particular, para las propiedades mecánicas de MOR y adhesión interna. Sin embargo, los porcentajes de absorción de humedad y variación de espesor en algunos casos no cumplieron con los valores mínimos aceptados por norma.

De acuerdo con los resultados obtenidos, el tablero homogéneo de partículas de $P$. patula con una densidad de $767 \mathrm{~kg}$ $\mathrm{m}^{-3}$ presentó las mejores características en cuanto a propiedades mecánicas de MOR, adhesión interna y mejor comportamiento en la absorción de humedad a 2 y 24 horas y variación de espesor a 2 horas.

Finalmente, los tableros aglomerados de partículas de $P$. patula homogéneos de una capa, encolados con urea-formaldehído y de ambas densidades poseen buena resistencia mecánica y adhesión interna, por lo que se recomienda su uso en partes internas de muebles y gabinetes, como material decorativo superpuesto sobre otros materiales, como recubrimiento interno de casas prefabricadas, entre otros usos; siempre y cuando el tablero preste su servicio en sitios con poca o nula exposición directa a la humedad. 


\section{REFERENCIAS}

Alma, M., H. Kalaycroglu, I. Bektas y A. Tutus. 2004. Properties of cotton carpel based particleboards. Industrial Crops and Products 2:141-149.

Blanchet, P., A. Cloutier y B. Rield, B. 2000. Particleboard made from hammer milled black spruce bark residues. Wood Science and Technology 34(1):11-19.

Bazyar, B., A. Tichi y H. Rangavar. 2011. Particleboard made from fast growing Aspen wood and old rail road ties. Australian Journal of Basic and Applied Sciences 5(8):548-553.

Cai, Z y R. Ross. 2010. Mechanical properties of wood-based composite materials. In: R. J. Ross, ed. Wood handbook-Wood as an engineering material. Centennial edition. General Technical Report FPL-GTR-190. Department of Agriculture, Forest Service, Forest Products Laboratory. Madison, Wisconsin, EUA. 12 p.

COVENIN (Comisión Venezolana de Normas Industriales). 1991. Norma venezolana para tableros de partículas de madera (provisional) COVENIN-847-91. Ministerio de Fomento. Caracas-Venezuela.

DIN (Deutsches Institut fur Normung). 1965a. Testing of wood chipboards; general requirements, sampling, evaluation. German National Standard. Standard DIN 52360.

DIN (Deutsches Institut fur Normung). 1965b. Testing of wood chipboards; determination of dimensions, raw density and moisture content. German National Standard. Standard DIN 52361. 2p.
DIN (Deutsches Institut fur Normung). 1965c. Testing of wood chipboards; bending test, determination of bending strength. German National Standard. Standard DIN 52362. 2p.

DIN (Deutsches Institut fur Normung). 1965d. Testing of wood chipboards; determination of variation in thickness due to moisture. German National Standard. Standard DIN 52364. $1 \mathrm{p}$.

DIN (Deutsches Institut fur Normung). 1965e. Testing of wood chipboards; determination of tensile strength vertical to the chipboard plane. German National Standard. Standard DIN 52365. $1 \mathrm{p}$.

DIN (Deutsches Institut fur Normung). 1976. Testing of wood; determination of density. German National Standard. Standard DIN 52182. 3 p.

DIN (Deutsches Institut fur Normung). 1981. Particle boards; flat pressed boards for general purposes; FPOboard. German National Standard. Standard DIN 68761.

Durán, J. 1981. Utilización de los aclareos de las especies de la plantación de Caparo para tableros aglomerados de partículas: Parte I, Teca. Universidad de Los Andes, Laboratorio Nacional de Productos Forestales. Mérida, Venezuela. $77 \mathrm{p}$.

Durán, J. 1982. Utilización de los aclareos de las especies de la plantación de Caparo para tableros aglomerados de partículas: Parte II, Gmelina. Universidad de Los Andes, Laboratorio Nacional de Productos Forestales. Mérida, Venezuela. $44 \mathrm{p}$.

Garay, D. 1997. Tableros aglomerados de partículas. Laboratorio Nacional de 
Productos Forestales, Facultad de Ciencias Forestales y Ambientales, Universidad de Los Andes. Mérida, Venezuela.

Garay, M., F. MacDonald, M. Acevedo, B. Calderón y J. Araya. 2009. Particleboard made with crop residues mixed with wood from Pinus radiata. BioResources 4(4):1396-1408.

Gatchell, G., B. Heebink y F. Hefty. 1966. Influence of component variables on the properties of particleboard for exterior use. Forest Products Journal 16(4):46-59.

Ginzel, C y W. Peraza. 1966. Tecnología de tableros de partículas. Instituto de Investigación y Experiencias. Madrid. $185 \mathrm{p}$.

Guler, C., Y. Copur y C. Tascioglu. 2008. The manufacture of particleboard using mixture of peanut hull (Arachis hypoqaeae L.) and European black pine (Pinus nigra Arnold) wood chip. Bioresource Technology 99(8): 2893-2897.

Halligan, A. 1970. A review of thickness swelling in particleboard. Wood Science and Technology 4(4):301-312.

Latibari, A y M. Roohnia. 2010. Potential of utilization of the residues from Poplar plantation for particleboard production in Iran. Journal of Forestry Research 21(4):503-508.

Lehmann, F. 1960. The effects of moisture content, board density and press temperature on the dimensional and strength properties of flat-pressed flakeboard. School Forestry, North Carolina State Univ., Raleigh, N.C. $106 \mathrm{pp}$.
Maloney, T. 1993. Modern particleboard and dry-process fiberboard manufacturing. Miller Freeman Inc., San Francisco, California, EUA. 686 p.

McNatt, J. 1973. Basic engineering properties of particleboard. Res. Pap. FPL-206. Madison, WI: U.S. Department of Agriculture, Forest Service, Forest Products Laboratory. $14 \mathrm{p}$.

Medina, A. 1975. Seminario sobre proceso de fabricación de tableros de partículas de madera. Facultad de Ciencias Forestales y Ambientales, Universidad de Los Andes. MéridaVenezuela.

Moreno, P., J. Durán, y D. Garay. 2002. Efecto de las Sales CCA sobre las propiedades físicas y mecánicas en tableros de partículas de pino caribe. Revista Forestal Latinoamericana 17 (1):1-34.

Moreno, P., J. Durán, D. Garay, S. Valero, T. Shakespeare y R. Nieto. 2005. Utilización de la madera de Fraxinus americana (fresno) en la fabricación de tableros de partículas. Revista Theoria 14(2):57-64.

Moreno, P., J. Durán, D. Garay y S. Valero. 2010. Utilización de tallos de Guadua angustifolia en la fabricación de tableros de partículas. Forest Systems 19(2):241-248.

Moslemi, A. 1974. Particleboard. Vol. I Materials. Southern Illinois University Press. 239 p.

Peredo, M y H. Deppe. 1984. Zur Verwendung von Biomasse bei der Herstellung von Holzspanplatten für das Bauwesen. Forstarchiv 55(2):65-70. 
Peredo, M. 1988. Fabricación de tableros de partículas para uso exterior. Bosque (Valdivia) 9(1):35-42.

Peredo, M y E. Torres. 1991. Fabricación de tableros de partículas con desechos industriales. Bosque (Valdivia) 12(1):49-57.

Pedieu, R., B. Riedl y A. Pichette. 2008. Physical and mechanical properties of panel based on outer bark particles of white birch: mixed panels with wood particles versus wood fibres. Maderas: Ciencia y Tecnología 10(3):195-206.

Poblete, H y M. Peredo. 1990. Tableros de desechos del debobinado de especies chilenas. Bosque (Valdivia) 11(2):45-58.

Poblete, H y J. Sánchez. 1991. Tableros con corteza de Pinus radiata. Bosque (Valdivia) 12(1):17-26.

Rocha, B., W. Lehmann y R. Sidney. 1974. How species and board densities affect properties of exotic hardwood particleboards. Forest Product Journal 24(12):37-45.

Roffael, E y W. Rauch. 1972. Influence of density on the swelling behavior of the phenolic resin bonded particleboards. Holz als Roh-und Werkstoff 30(1):178-181.

Stark, N., Z. Cai y C. Carll. 2010. Woodbased composite materials panel products, glued-laminated timber, structural composite lumber, and wood-nonwood composite materials. In: R.J. Ross, ed. Wood handbookWood as an engineering material. Centennial edition. General Technical Report FPL-GTR-190. Department of Agriculture, Forest Service, Forest Products Laboratory. Madison, Wisconsin, EUA p:1-28.

Suchsland, O y H. Xu. 1989. A Simulation of the horizontal density distribution in a flake-board. Forest Products Journal 39 (5):29-33.

Van Niekerk, I y A. Pizzi. 1994. Characteristic industrial technology for exterior Eucalyptus particleboard. Holz als Roh-und Werkstoff 52(2):109-112.

Zheng, Y., R. Pan, B. Jenkins y S. Blunk. 2006. Properties of medium-density particleboard from saline Athel wood. Industrial Crops and Products 23(3):318-326. residuos industriales de la madera Pinus patula. Madera y Bosques 19(3):65-80. 